\title{
Effects of Thyroperoxidase Antibody and Thyroglobulin Antibody on Maternal and Neonatal Outcomes in Pregnant Women
}

Authors

Li Wang ${ }^{1}$, Yi Tang ${ }^{1}$, Yi Yuan ${ }^{1}$, Lin Yu1 , Bai Jin ${ }^{2}$, Jun Xia ${ }^{3}$, Qingxin Yuan ${ }^{1}$

Affiliations

1 Department of Endocrinology and Metabolism, Affiliated Hospital, Nanjing Medical University, Nanjing, China

2 Department of Obstetrics, Affiliated Hospital, Nanjing Medical University, Nanjing, China

3 Department of Endocrinology and Metabolism, Jiangsu Shengze Hospital, Nanjing Medical University, Suzhou, China

Key words

thyroperoxidase antibody, thyroglobulin antibody, pregnancy, postpartum thyroiditis (PPT)

received 25.09.2021

accepted after revision $\quad 27.12 .2021$

Bibliography

Horm Metab Res 2022; 54: 76-83

DOI 10.1055/a-1731-7572

ISSN 0018-5043

(c) 2022. Thieme. All rights reserved.

Georg Thieme Verlag, Rüdigerstraße 14,

70469 Stuttgart, Germany

Correspondence

Qingxin Yuan

Department of Endocrinology and Metabolism

The First Affiliated Hospital of Nanjing Medical University

210029 Nanjing

China

Tel.: + 8613851654889

yqx@njmu.edu.cn

\section{ABSTRACT}

The aim of the study was to evaluate the effects of thyroperoxidase antibody (TPOAb) and thyroglobulin antibody (TgAb) on maternal and neonatal adverse outcomes in pregnant women. A total of 296 singleton pregnant women were classified into four groups according to the thyroid auto-antibody in the first trimester. Finally, there were 97 women in TPOAb positive group (TPOAb + /TgAb-), 35 in TgAb positive group (TPOAb-/ $\mathrm{TgAb}+$ ), 85 in TPOAb and TgAb positive group (TPOAb + $\mathrm{TgAb}+$ ), and 79 in TPOAb and TgAb negative group (TPOAb-/

Li Wang and Yi Tang contributed equally to this work and should be regarded as joint first authors
TgAb-). Thyroid function, TPOAb, and TgAb were checked during pregnancy and followed up at 6 weeks, 3 months, 6 months, 9 months, and 12 months postpartum. Levothyroxine sodium tablets could be taken to maintain euthyroid antepartum. Thyroid function of women with postpartum thyroiditis (PPT) were followed up at 2 and 3 years postpartum. We observed the incidence of PPT, premature rupture of membranes (PROM), placental abruption, placenta previa, polyhydramnios, oligohydramnios, postpartum hemorrhage, preterm birth, and low birth Weight in the four groups. $19.93 \%$ of the women had PPT. The incidence of PPT in TPOAb + /TgAb-, TPOAb-/TgAb + , TPO$\mathrm{Ab}+/ \mathrm{TgAb}+$ groups was significantly higher than that in TPO$\mathrm{Ab}-/ \mathrm{TgAb}-$ group, respectively (16.49 vs. $6.33 \%, 22.86$ vs. $6.33 \%, 35.29$ vs. $6.33 \%, p<0.05)$. The incidence of PPT in $\mathrm{TPOAb}+/ \mathrm{TgAb}+$ group was significantly higher than that in $\mathrm{TPOAb}+/ \mathrm{TgAb}-$ group (35.29 vs. $16.49 \%, \mathrm{p}<0.01)$. PPT occurred as early as 6 weeks postpartum, but mainly at 3 and 6 months postpartum in the four groups $(62.50 \%, 75.00 \%$, $70.00 \%, 80.00 \%$ ). All PPT in TPOAb-/TgAb- group occurred within 6 months postpartum, while it was found at 9 months or 12 months postpartum in other three groups. There was no classical form of PPT in TPOAb-/TgAb- group, while in the other three groups, all three types (classical form, isolated thyrotoxicosis, isolated hypothyroidism) existed. At 2 years postpartum of the women with PPT, the rate of euthyroidism in $T P O A b+/ T g A b+$ group was significantly lower than that in TPOAb-/TgAb- group $(\mathrm{p}<0.05)$. At 3 years postpartum of the women with PPT, the rate of euthyroidism in TPOAb + $/ \mathrm{TgAb}-$, $\mathrm{TPOAb}-/ \mathrm{TgAb}+$, and TPOAb $+/ \mathrm{TgAb}+$ groups were significantly lower than that in TPOAb-/TgAb- group $(p<0.05)$. The values of TPOAb and $\mathrm{TgAb}$ postpartum were significantly higher than those during pregnancy $(p<0.05)$. The incidence of $\mathrm{PROM}$ in TPOAb + $\mathrm{TgAb}$ - group was significantly higher than that in TPOAb-/TgAb-group (32.99 vs. $17.72 \%, \mathrm{p}<0.05$ ). The binary logistic regression for PPT showed that the OR value of TPOAb was $2.263(95 \% \mathrm{Cl} 1.142-4.483, \mathrm{p}=0.019)$. The OR value of $\operatorname{TgAb}$ was $3.112(95 \% \mathrm{Cl} 1.700-5.697, \mathrm{p}=0.000)$. In conclusion, pregnant women with positive thyroid auto-antibodies had an increased risk of PPT and a reduced rate of euthyroidism at 2 and 3 years postpartum. TPOAb is associated with the incidence of PROM. Both of TPOAb and TgAb were independent risk factors for PPT. TgAb deserves more attention when studying autoimmune thyroid disease (AITD) combined with pregnancy. 


\section{Introduction}

Autoimmune thyroid disease (AITD) is one of the most common diseases that lead to thyroid dysfunction of women in reproductive age. It could not only cause the thyroid dysfunction of pregnant women, but also result in adverse outcomes for both mother and child [1]. Positive thyroid auto-antibodies are symbols of autoimmune thyroid disease, which include thyroperoxidase antibody (TPOAb), thyroglobulin antibody (TgAb), thyroid stimulating hormone receptor antibody (TRAb), and sodium/iodide symporter (NIS) antibody. TRAb is the specific marker of Graves disease (GD), while TPOAb and TgAb are responsible for Hashimoto thyroiditis (HT).

TPO (thyroid peroxidase) catalyzes the iodination of thyroglobulin tyrosine during the biosynthesis of T3 and T4 and is the main antigen of thyromicrosome (TM). TPOAb works directly against TPO and may damage thyroid cells, leading to insufficient hormone secretion through cell-mediated and antibody-dependent cytotoxic effects [2]. After binding to thyroglobulin ( $\mathrm{Tg}), \mathrm{TgAb}$ can be bound to the Fc receptor, activate NK cells and attack target cell, resulting in thyroid cells destruction. TgAb also affects the uptake and processing of $\mathrm{Tg}$, and catalyzes the hydrolysis of $\mathrm{Tg}$, thus affecting non-significant T-cell antigen determinants of autoimmune response and resulting in the exacerbation of AITD [2].

According to the epidemiology, the prevalence of TPOAb and (or) $\mathrm{TgAb}$ in the general population of reproductive age is $10-20 \%$, of which the positive rate of TPOAb is $10-13 \%$ and the positive rate of $\mathrm{TgAb}$ is $9-14 \%$ [3]. A large number of studies have reported that TPOAb in pregnant women has relationship with adverse maternal and neonatal outcomes, such as miscarriage, preterm delivery, placental abruption, premature rupture of membranes (PROM), low birth weight (LBW) and neonatal respiratory distress syndrome. In a prospective study of 3959 women in the first trimester of pregnancy, Negro et al. reported significantly increased risk of preterm delivery in TPOAb positive women and observed a higher incidence of neonatal respiratory distress syndrome in TPOAb positive women [4]. In addition, TPOAb positivity also has long-term effects on maternal health postpartum and intellectual development of the offspring. An association between TPOAb positivity during pregnancy and postpartum depression was observed by Kuijpens et al. [5]. Pop et al. assessed the IQ of the offspring of TPOAb positive women and TPOAb negative women at the 32th week of the gestation and found that the offspring of TPOAb positive women had an IQ score 10.5 points lower than that of the TPOAb negative group [6]. Postpartum thyroiditis (PPT) is a thyroid dysfunction syndrome that occurs within 1 year after delivery. Typical clinical manifestations are divided into three stages, initial thyrotoxicosis followed by hypothyroidism, and a recovery to euthyroid state. Some patients only present isolated hyperthyroidism or hypothyroidism. Permanent hypothyroidism remains in $20 \%$ of them. Our previous study showed that TPOAb positive pregnant women had an increased incidence of hyperhydramnios and PPT [7]. In the past few decades, researchers have paid much attention to TPOAb when studying pregnant outcomes, neglecting $\mathrm{TgAb}$ at the same time. Besides, compared with TPOAb, TgAb is not usually involved in routine pregnancy tests in many areas. One study carried by Williams et al. evaluated the neurocognitive ability of 43 children aged 5.5-years who were born at term. Children with $\mathrm{TgAb}$ positive mothers had lower sensory performance and exercise scores [8]. The importance of $\mathrm{TgAb}$ in pregnant women with AITD needs further study.

Hence, this study was to observe the effects of TPOAb and/or TgAb during pregnancy on adverse maternal and neonatal outcomes of pregnant women in an iodine-rich area in China. Compared with previous study [7], we took $\mathrm{TgAb}$ into account. Moreover, we followed up the changes of thyroid function in patients with PPT to 3 years postpartum.

\section{Subjects and Methods}

\section{Subjects}

Subjects were selected from single pregnant women who received routine prenatal examinations in the outpatient department of jiangsu Province Hospital from February 2015 to February 2021. Pregnant women with pre-pregnancy thyroid dysfunction, family history of thyroid disease, recognized autoimmune disease, history of taking medications that affect thyroid function, diseases related to heart, liver, kidney, etc., and TRAb positive were excluded from this study. According to the thyroid auto-antibody in the first trimester, 97 women were classified as TPOAb positive alone (TPOAb + /TgAb-), 35 as TgAb positive alone (TPOAb-/TgAb +), 85 as TPOAb and TgAb positive (TPOAb $+/ \mathrm{TgAb}+)$, and 79 as TPOAb and TgAb negative (TPOAb-/TgAb-), respectively ( $>$ Fig. $\mathbf{1}$ ).

\section{Data collection}

We inquired the subjects about their age, parity, and history of spontaneous abortion. Height, weight, systolic blood pressure and diastolic pressure were obtained from a 12-week gestation record of the pregnant women. Body mass index was calculated by dividing your weight $(\mathrm{kg})$ by the square of your height $(\mathrm{m})$. All participants underwent a $75 \mathrm{~g}$ oral glucose tolerance test at 24-28 weeks of gestation. Fasting blood glucose (FBG), 1 hour postprandial blood glucose (PBG1h) and 2 hours postprandial blood glucose (PBG2h) were recorded. During the whole pregnancy, these women were tested for thyroid function (TSH, FT3, FT4) and urine iodine concentration (UIC) every 4 weeks. Levothyroxine sodium tablets could be taken to maintain euthyroid antepartum and were

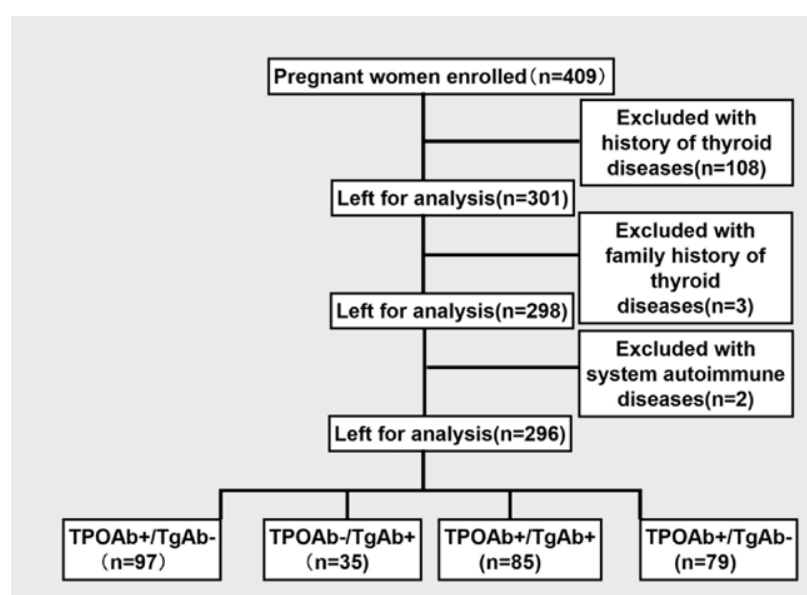

Fig. 1 The flow diagram of the study process. 
stopped taking postpartum. Thyroid function was followed up at 6 weeks, 3 months, 6 months, 9 months and 12 months postpartum. Thyroid function of women with PPT were followed up at 2 and 3 years postpartum. The newborns' sex, height, weight, and Apgar score were obtained from the obstetric records at the time of delivery. The time and mode of delivery and adverse outcomes were obtained from the obstetric discharge records.

\section{Measurements}

An amount of $3 \mathrm{ml}$ of blood sample was taken from each subject after an overnight fast. TSH, FT4, TPOAb and TgAb were measured by chemiluminescence immunoassay (Roche Cobas 6000, ZB/ GEM1815-2010, Mannheim, Germany). The coefficient of variation (CV) for TSH and FT4 was 5.3 and $7.8 \%$ respectively, and the intra-assay CV was 6.4 and $7.1 \%$, respectively. UIC was determined by arsenic and cerium catalytic spectrophotometry.

We used the Roche kit's reference range in ATA Guidelines for the management of thyroid diseases during pregnancy (TSH 0.09$4.52 \mathrm{mIU} / \mathrm{l}$, first trimester; $0.45-4.32 \mathrm{mIU} / \mathrm{I}$, second trimester; $0.30-4.98 \mathrm{mIU} / \mathrm{l}$, third trimester and FT4 13.15-20.78 pmol/l, first trimester; 9.77-18.89 pmol/l, second trimester; 9.04-15.22 $\mathrm{pmol} / \mathrm{l}$, third trimester). The titer of TPOAb $>34 \mathrm{IU} / \mathrm{ml}$ was considered as being positive. The titer of $\mathrm{TgAb}>115 \mathrm{IU} / \mathrm{ml}$ was considered as being positive.

\section{Definition of abnormal maternal and neonatal outcomes}

\begin{tabular}{|c|c|}
\hline Cesarean Section(CS) & $\begin{array}{l}\text { CS is recorded by the midwives who participates } \\
\text { in labor }\end{array}$ \\
\hline Placenta previa & $\begin{array}{l}\text { The placenta grows in the lowest part of the } \\
\text { womb (uterus) and covered all or part of the } \\
\text { opening to the cervix }\end{array}$ \\
\hline Placental abruption & $\begin{array}{l}\text { The normally located placenta completely or } \\
\text { partially separates from the uterine site before } \\
\text { the birth of fetus }\end{array}$ \\
\hline PROM & $\begin{array}{l}\text { The membrane of the amniotic sac and chorion } \\
\text { is ruptured at least } 1 \mathrm{~h} \text { before the onset of labor }\end{array}$ \\
\hline $\begin{array}{l}\text { Postpartum } \\
\text { hemorrhage(PPH) }\end{array}$ & $\begin{array}{l}\text { More than } 500 \mathrm{ml} \text { or } 1000 \mathrm{ml} \text { of blood loss } \\
\text { within the first } 24 \text { hours after childbirth }\end{array}$ \\
\hline Polyhydramnios & The amount of amniotic fluid exceeds $2000 \mathrm{ml}$ \\
\hline Oligohydramnios & The amount of amniotic fluid is less than $400 \mathrm{ml}$ \\
\hline PPT & $\begin{array}{l}\text { Thyroid dysfunction occurs one year after } \\
\text { delivery, including classical form, isolated } \\
\text { thyrotoxicosis, isolated hypothyroidism }\end{array}$ \\
\hline Preterm birth & The delivery occurs before 37 weeks' gestation \\
\hline Fetal distress & $\begin{array}{l}\text { The symptoms of fetal hypoxia, including fetal } \\
\text { bradycardia, serious variable decelerations and } \\
\text { lasting late decelerations occurs }\end{array}$ \\
\hline LBW & Fetal birth weight $<2500 \mathrm{~g}$ \\
\hline Macrosomia & Fetal birth weight $>4000 \mathrm{~g}$ \\
\hline Neonatal diseases & $\begin{array}{l}\text { Including neonatal hemorrhage, neonatal } \\
\text { infections, neonatal defects, neonatal jaundice, } \\
\text { neonatal thrombocytopenia }\end{array}$ \\
\hline
\end{tabular}

\section{Statistical analysis}

SPSS 25.0 software was used to analyze the data. First, the normality and homogeneity of continuous variables were examined. If the data were normally distributed and had uniform variances, data were expressed as means \pm standard deviation and one-way ANOVA was used to compare separate groups; Brown-Forsythe analysis was used if the data did not have uniform variances. Variables that were not normally distributed (e. g., urinary iodine concentration, Tope value, TgAb value) were expressed as median with interquartile. Comparisons between four groups were performed by using Kruskal-Wallis $\mathrm{H}$-test, and comparisons between two groups were performed by using Mann-Whitney M-test. TPOAb or TgAb levels were presented as scatterplots marked with 25th, median, 75th percentile values. The abnormal maternal and neonatal outcomes were presented as $n(\%)$ and chi-square test was used. But if the theoretical frequency was 0 or the numbers of squares with theoretical frequency less than 5 accounted for more than $20 \%$, Fisher exact test was replaced. Binary logistic regression analysis was expressed as OR $(95 \% \mathrm{Cl})$. A p-value $<0.05$ was considered significantly.

\section{Ethics Approval}

This study was approved by the Ethics Committee of the First Affiliated Hospital of Nanjing Medical University. All participants completed a written informed consent form.

\section{Results}

\section{Population Characteristics of pregnant women}

- Table 1 shows the baseline characteristics of the study population. A total of 296 pregnant women were included and classified into four groups, 97 (32.77\%) women in tope positive group (TPOAb + / TgAb-), 35(11.82\%) women in TgAb positive group (TPOAb-/TgAb + ), 85 (28.72\%) women in TPOAb and TgAb positive group (TPOAb $+/ \mathrm{TgAb}+)$, and $79(26.69 \%)$ women in TPOAb and TgAb negative group (TPOAb-/TgAb-). There were no significant differences in age, BMI, parity, history of spontaneous abortion, blood pressure, and blood glucose among four groups $(p>0.05)$.

\section{The level of antibodies antepartum and postpartum in TPOAb positive group, $\mathrm{TgAb}$ positive group, and TPOAb-/TgAb- groups}

The values of TPOAb in TPOAb + /TgAb- and TPOAb + TgAb + group (a) and TgAb in TPOAb-/TgAb + TPOAb + /TgAb + group (b) postpartum were significantly higher than those during pregnancy $(\mathrm{p}<0.05)$ ( Fig. 2).

In the TPOAb-/TgAb-group, no significant differences were found in the values of antibodies between antepartum and postpartum ( $p>0.05)$ ( $\vdash$ Fig. 3).

\section{The incidence of PPT in the four group}

A total of $19.93 \%$ of the women had PPT. The incidence of PPT in TPOAb + /TgAb-, TPOAb-/TgAb +, TPOAb + /TgAb + groups was significantly higher than that in TPOAb-/TgAb-group, respectively ( 16.49 vs. $6.33 \%, 22.86$ vs. $6.33 \%, 35.29$ vs. $6.33 \%, p<0.05$ ). The incidence of PPT in TPOAb $+/ \mathrm{TgAb}+$ group was significantly higher than that in TPOAb + /TgAb- group (35.29 vs. $16.49 \%, p<0.01$ ). 
PPT was found as early as 6 weeks postpartum, but the most onset time was between 3 months and 6 months postpartum in four groups (62.50\%, 75.00\%, 70.00\%, 80.00\%). All PPT in TPOAb-I $\mathrm{TgAb}$ - group occurred within 6 months postpartum, while it may have occurred at 9 months or 12 months postpartum in other three groups. There is no classical form of PPT in TPOAb-/TgAb-group, while in the other three groups, all three types (classical form, isolated thyrotoxicosis, isolated hypothyroidism) existed ( $\triangleright$ Table 2).

Binary logistic regression was performed on PPT among these four groups. The OR value of TPOAb was 2.263 (95\% Cl 1.142-

- Table 1 Population characteristics of the four groups.

\begin{tabular}{|c|c|c|c|c|c|c|}
\hline & $\mathrm{TPOAb}+/ \mathrm{TgAb}-(\mathrm{n}=97)$ & $\operatorname{TPOAb} / \mathrm{TgAb}+(n=35)$ & $\mathrm{TPOAb}+/ \mathrm{TgAb}+(\mathrm{n}=85)$ & TPOAb-/TgAb- $(n=79)$ & $\mathrm{X}^{2} / \mathrm{F}$ & $\mathbf{p}$ \\
\hline \multicolumn{7}{|l|}{ Maternal } \\
\hline Age (years) & $30.31 \pm 4.89$ & $31.23 \pm 3.99$ & $29.85 \pm 4.29$ & $29.23 \pm 3.64$ & 2.046 & 0.11 \\
\hline BMI $\left(\mathrm{kg} / \mathrm{m}^{2}\right)$ & $21.22 \pm 3.16$ & $22.18 \pm 3.42$ & $21.08 \pm 2.63$ & $21.71 \pm 4.38$ & 1.136 & 0.34 \\
\hline Parity & & & & & 3.271 & 0.35 \\
\hline Nulliparity & $72(74.23 \%)$ & $26(74.29 \%)$ & $67(78.82 \%)$ & $67(84.81 \%)$ & & \\
\hline Multiparity & $25(25.77 \%)$ & $9(25.71 \%)$ & $18(21.18 \%)$ & $12(15.19 \%)$ & & \\
\hline $\begin{array}{l}\text { History of spontaneous } \\
\text { abortion }\end{array}$ & $48(49.48 \%)$ & $14(40.00 \%)$ & $40(47.06 \%)$ & $31(39.24 \%)$ & 2.346 & 0.50 \\
\hline \multicolumn{7}{|l|}{ Blood pressure } \\
\hline Systolic pressure $(\mathrm{mmHg})$ & $117.90 \pm 8.89$ & $115.47 \pm 9.55$ & $118.40 \pm 9.94$ & $118.61 \pm 10.45$ & 0.923 & 0.43 \\
\hline Diastolic Pressure $(\mathrm{mmHg})$ & $75.74 \pm 7.06$ & $74.76 \pm 5.72$ & $75.48 \pm 8.33$ & $77.08 \pm 8.23$ & 0.961 & 0.41 \\
\hline $\mathrm{FBG}(\mathrm{mmol} / \mathrm{l})$ & $4.50 \pm 0.41$ & $4.61 \pm 0.65$ & $4.62 \pm 0.68$ & $4.61 \pm 0.55$ & 0.787 & 0.50 \\
\hline PBG1h (mmol/l) & $8.02 \pm 1.96$ & $8.44 \pm 1.48$ & $8.29 \pm 1.76$ & $7.88 \pm 1.54$ & 0.913 & 0.44 \\
\hline PBG2h (mmol/l) & $7.17 \pm 1.54$ & $7.22 \pm 1.35$ & $7.34 \pm 1.32$ & $6.75 \pm 0.92$ & 1.995 & 0.12 \\
\hline MUIC $(\mu \mathrm{g} / \mathrm{l})$ & $200(100,250.30)$ & $200(100,250)$ & $200(93.75,300)$ & $200(100,300)$ & 0.250 & 0.97 \\
\hline
\end{tabular}

TPOAb: Thyroid peroxidase antibody; TgAb: Thyroglobulin antibody; BMI: Body mass index; MUIC: Median urine iodine concentration; FBG: Fasting blood glucose; PBG1h: 1h Plasma blood glucose; PBG2h: 2h Plasma blood glucose.
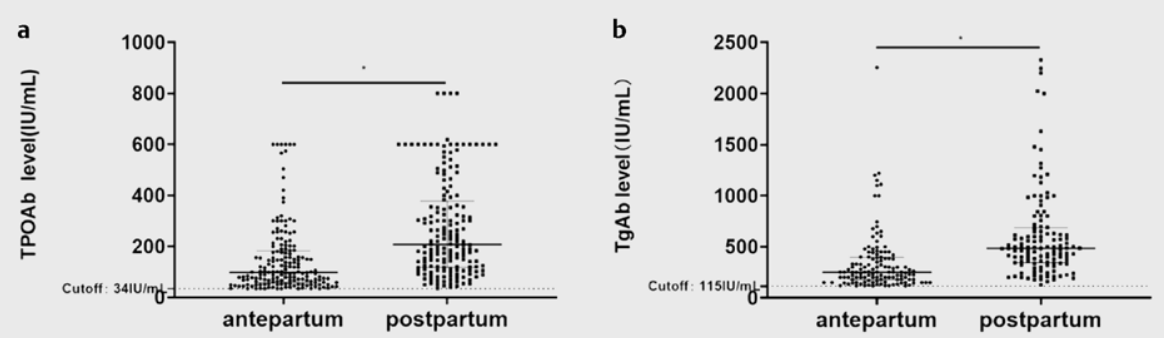

- Fig. 2 The level of TPOAb in TPOAb +/TgAb- and TPOAb +/TgAb + group. a: and TgAb in TPOAb-/TgAb + and TPOAb +/TgAb + group; b: antepartum and postpartum.
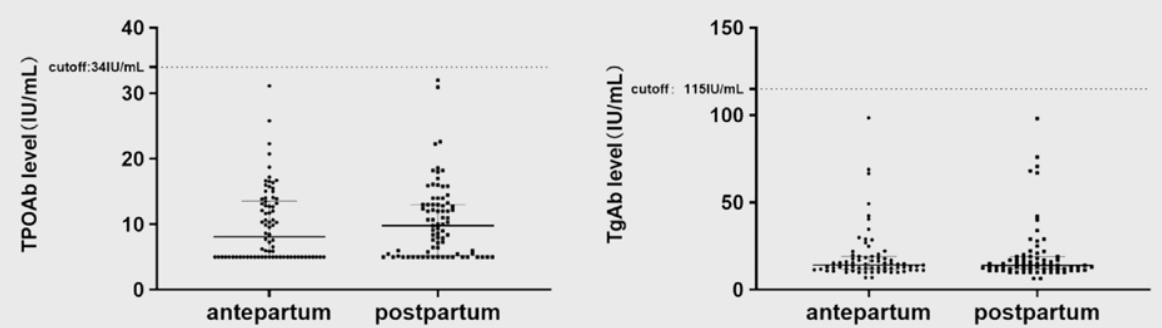

- Fig. 3 The level of antibodies antepartum and postpartum in TPOAb-/TgAb- group. 
> Table 2 The onset time and presenting form of PPT among four groups.

\begin{tabular}{|c|c|c|c|c|c|c|}
\hline & $\begin{array}{l}\text { TPOAb + I } \\
\text { TgAb- }(n=97)\end{array}$ & $\begin{array}{l}\text { TPOAb-I } \\
\operatorname{TgAb}+(n=35)\end{array}$ & $\begin{array}{l}\operatorname{TPOAb}+\text { I } \\
\operatorname{TgAb}+(n=85)\end{array}$ & $\begin{array}{l}\text { TPOAb-I } \\
\text { TgAb- }(n=79)\end{array}$ & $x^{2}$ & $\mathbf{p}$ \\
\hline PPT, n (\%) & $16(16.49 \%)^{*}$ a & $8(22.86 \%)^{*}$ & $30(35.29 \%)^{*}$ & $5(6.33 \%)$ & 22.634 & 0.00 \\
\hline \multicolumn{7}{|l|}{ Postpartum time of onset } \\
\hline 6 weeks postpartum, n (\%) & $4(25.00 \%)$ & $1(12.50 \%)$ & $7(23.33 \%)$ & $1(20 \%)$ & 0.600 & 0.92 \\
\hline 3 months postpartum, n (\%) & $4(25.00 \%)$ & $3(37.50 \%)$ & $12(40.00 \%)$ & $2(40 \%)$ & 1.271 & 0.77 \\
\hline 6 months postpartum, n (\%) & $6(37.50 \%)$ & $3(37.50 \%)$ & $9(30.00 \%)$ & $2(40 \%)$ & 0.737 & 0.88 \\
\hline 9 months postpartum, n (\%) & $2(12.50 \%)$ & $1(12.50 \%)$ & $1(3.33 \%)$ & $0(0 \%)$ & 2.506 & 0.47 \\
\hline 12 months postpartum, n (\%) & $0(0 \%)$ & $0(0 \%)$ & $1(3.33 \%)$ & $0(0 \%)$ & 2.338 & 1.00 \\
\hline \multicolumn{7}{|l|}{ Presenting form } \\
\hline Classical form, n (\%) & $6(37.50 \%)$ & $2(25.00 \%)$ & $9(30.00 \%)$ & $0(\%)$ & 2.374 & 0.53 \\
\hline Isolated thyrotoxicosis, $\mathrm{n}(\%)$ & $6(37.50 \%)$ & $3(37.50 \%)$ & $10(33.33 \%)$ & $1(20 \%)$ & 0.639 & 0.97 \\
\hline Isolated hypothyroidism, n (\%) & $4(25.00 \%)$ & $3(37.50 \%)$ & $11(36.67 \%)$ & $4(80 \%)$ & 4.610 & 0.20 \\
\hline
\end{tabular}

PPT: Postpartum thyroiditis; " $\mathrm{p}<0.05$, compared with TPOAb-/TgAb- group; a $\mathrm{p}<0.01$, the percentage of PPT between TPOAb $+/ \mathrm{TgAb}-$ group and $\mathrm{TPOAb}+/ \mathrm{TgAb}+$ group .

4.483, $\mathrm{p}=0.019)$. The OR value of TgAb was $3.112(95 \% \mathrm{Cl} 1.700-$ $5.797, \mathrm{p}=0.000)$. It means that TPOAb positivity has 2.263 times the risk of developing PPT compared with TPOAb negativity. Similarly, TgAb positivity has 3.112 times the risk of developing PPT compared with $\mathrm{TgAb}$ negativity ( $\triangleright$ Fig. 4).

\section{The thyroid function of women with previous diagnosis of PPT at 2 and 3 years postpartum}

A total of 59 women with previous diagnosis of PPT were followed up at 2 years postpartum. Unfortunately, 22 cases lost to follow-up and 37 were left eventually, including 9 in TPOAb $+/ \mathrm{TgAb}$ - group, 7 in TPOAb-/TgAb + group, 19 in TPOAb + TgAb + group, and 2 in TPOAb-/TgAb- group.

At 2 years postpartum of the women with PPT, the rate of euthyroidism in TPOAb $+/ \mathrm{TgAb}+$ group was significantly lower than that in TPOAb-/TgAb-group ( $p<0.05$ ). Hypothyroidism accounted for the most in auto-antibody positive group, especially in

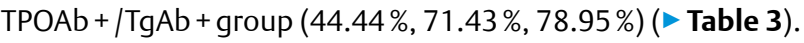

At 3 years postpartum of the women with PPT, the rate of euthyroidism in TPOAb + /TgAb-, TPOAb-/TgAb +, TPOAb + $\mathrm{TgAb}+$ groups were significantly lower than that in TPOAb-/TgAbgroup ( $p<0.05)$. Hypothyroidism accounted for the most in auto-antibody positive group (77.78\%, 85.71\%, 84.21\%) ( Table 4).

\section{Adverse maternal outcomes among four groups}

We compared adverse maternal outcomes of these four groups in pairs and found that the incidence of PROM in TPOAb + TgAbgroup was significantly higher than that in TPOAb-/TgAb- group ( 32.99 vs. $17.72 \%$, p <0.05). No significant difference was shown in the gestation time, mode of delivery, placenta previa, placental abruption, polyhydramnios, oligohydramnios, and PPH after pairwise comparisons $(p>0.05)$ ( $>$ Table 5).

\section{Adverse neonatal outcomes among four groups}

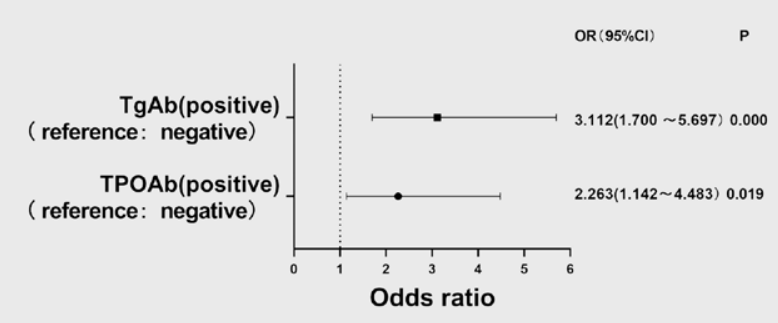

- Fig. 4 The binary logistic regression for PPT.

There were no significant differences in neonatal outcomes including sex, height, weight, and Apgar score at 1 and 5 minutes in the four groups $(p>0.05)$. No significant difference was shown in the incidence of preterm birth, fetal distress, LBW, neonatal disease, and macrosomia $(p>0.05)(\triangleright$ Table 6$)$.

\section{Discussion}

In this study, we investigated the effects of thyroid autoantibodies $\mathrm{TPOAb} / \mathrm{TgAb}$ on adverse pregnancy outcomes and thyroid function in women postpartum.

Previous researches have shown that PPT is a thyroid dysfunction after delivery in women who were euthyroid prior to pregnancy as a result of an immune "rebound" as a result of an immune "rebound" affected by the relative immunosuppression of pregnancy, with the prevalence of $1-22 \%$ [9]. Personal history of type 1 diabetes mellitus, systemic lupus erythematosus(SLE) or gestational diabetes mellitus, family history of thyroid disease, and the presence of TPOAb are reported to be risk factors for PPT [10]. In our study, $19.93 \%$ of the women had PPT. The incidence of PPT in TPOAb + / 
- Table 3 The thyroid function of women with previous diagnosis of PPT at 2 years postpartum.

\begin{tabular}{|c|c|c|c|c|c|c|}
\hline & $\operatorname{TPOAb}+/ \operatorname{TgAb}-(n=9)$ & TPOAb- $/ \mathrm{TgAb}+(n=7)$ & $\operatorname{TPOAb}+/ \operatorname{TgAb}+(n=19)$ & TPOAb-/TgAb- $(n=2)$ & $x^{2}$ & $\mathbf{p}$ \\
\hline Hyperthyroidism, n (\%) & $3(33.33 \%)$ & $1(14.29 \%)$ & $3(15.79 \%)$ & $0(0 \%)$ & 1.730 & 0.69 \\
\hline Hypothyroidism, n (\%) & $4(44.44 \%)$ & $5(71.43 \%)^{*}$ & $15(78.95 \%)$ & $0(0 \%)$ & 6.454 & 0.07 \\
\hline Euthyroidism, n (\%) & $2(22.22 \%)$ & $1(14.29 \%)$ & $1(5.26 \%)^{*}$ & $2(100 \%)$ & 8.675 & 0.02 \\
\hline
\end{tabular}

- Table 4 The thyroid function of women with previous diagnosis of PPT at 3 years postpartum.

\begin{tabular}{|c|c|c|c|c|c|c|}
\hline & $\operatorname{TPOAb}+/ \operatorname{TgAb}-(n=9)$ & TPOAb-/TgAb + $(n=7)$ & $\operatorname{TPOAb}+/ \operatorname{TgAb}+(\mathrm{n}=19)$ & TPOAb-/TgAb- $(n=2)$ & $x^{2}$ & $\mathbf{p}$ \\
\hline Hyperthyroidism, n (\%) & $1(11.11 \%)$ & $1(14.29 \%)$ & $2(10.53 \%)$ & $0(0 \%)$ & 0.960 & 1.00 \\
\hline Hypothyroidism, n (\%) & $7(77.78 \%)$ & $6(85.71 \%)$ & $16(84.21 \%)$ & $0(0 \%)$ & 5.768 & 0.09 \\
\hline Euthyroidism, n (\%) & $1(11.11 \%)^{*}$ & $0(0 \%)^{*}$ & $1(5.26 \%)^{*}$ & $2(100 \%)$ & 9.247 & 0.02 \\
\hline
\end{tabular}

- Table 5 Maternal outcomes among four groups.

\begin{tabular}{|c|c|c|c|c|}
\hline Pregnancy outcomes & $\operatorname{TPOAb}+/ \operatorname{TgAb}-(n=97)$ & TPOAb-/TgAb + $(n=35)$ & $\operatorname{TPOAb}+/ \operatorname{TgAb}+(n=85)$ & TPOAb-/TgAb- $(n=79)$ \\
\hline Gestational age at delivery (weeks) & $39.18 \pm 1.66$ & $39.31 \pm 1.21$ & $39.49 \pm 1.42$ & $39.19 \pm 1.78$ \\
\hline \multicolumn{5}{|l|}{ Mode of delivery } \\
\hline Vaginal & $58(59.79 \%)$ & $18(51.43 \%)$ & $46(54.12 \%)$ & $46(58.23 \%)$ \\
\hline Cesarean & $39(40.21 \%)$ & $17(48.57 \%)$ & $39(45.88 \%)$ & $33(41.77 \%)$ \\
\hline Placenta previa & $1(1.03 \%)$ & $0(0 \%)$ & $3(3.53 \%)$ & $1(1.27 \%)$ \\
\hline Placental abruption & $1(1.03 \%)$ & $0(0 \%)$ & $0(0 \%)$ & $0(0 \%)$ \\
\hline PROM & $32(32.99 \%)^{*}$ & $10(28.57 \%)$ & $22(25.88 \%)$ & $14(17.72 \%)$ \\
\hline Polyhydramnios & $4(4.12 \%)$ & $1(2.86 \%)$ & $1(1.18 \%)$ & $3(3.80 \%)$ \\
\hline Oligohydramnios & $7(7.22 \%)$ & $2(5.71 \%)$ & $5(5.88 \%)$ & $4(5.06 \%)$ \\
\hline PPH & $7(7.22 \%)$ & $2(5.71 \%)$ & $9(10.59 \%)$ & $5(6.33 \%)$ \\
\hline
\end{tabular}

PROM: Premature rupture of membranes; PPH: Postpartum hemorrhage; " $\mathrm{p}<0.05$, compared with TPOAb-/TgAb- group.

TgAb-, TPOAb-/TgAb + , TPOAb + /TgAb + groups were significantly higher than that in TPOAb-/TgAb-group. The correlation between TPOAb and PPT has been highlighted by prior research [11]. Through attacking different antigenic determinants of thyroglobulin located in the thyroid follicular colloid, $\mathrm{TgAb}$ is closely related to the synthesis and secretion of thyroid hormone [12]. Despite the fact that whether TgAb acts as a risk factor for PPT remains inconclusive, the prevalence of PPT in TgAb positive group was significantly higher compared with the control group, suggesting that the role of TgAb playing in the pathogenesis of PPT may have been underestimated before. Furthermore, binary logistic regression was performed among these four groups and showed that the OR value of TPOAb and TgAb were 2.263 and 3.112, separately, emphasizing both TPOAb and $\mathrm{TgAb}$ as risk factors for PPT. It is generally considered that during pregnancy, serum TPOAb and TgAb levels are reduced as a result of immune tolerance, but return to or even higher than pre-pregnancy levels after postpartum, thus leading to increased thyroid damage [13]. Besides, our study demonstrated that the incidence of PPT in TPOAb + /TgAb + group was significantly higher than that in single TPOAb or $\mathrm{TgAb}$ positive group. The cross-reactivity between TPOAb and TgAb are responsible for the origin of thyroid autoimmunity, providing possible explanation 
- Table 6 Neonatal outcomes among four groups.

\begin{tabular}{|l|l|l|}
\hline Neonatal outcomes & TPOAb $+/ \mathbf{T A} \mathbf{A b}-(\mathbf{n}=\mathbf{9 7})$ & TPOAb-/TgAb + (n \\
\hline Sex of neonate $($ boy, $\%)$ & $56(57.73 \%)$ & $19(54.29 \%)$ \\
\hline Neonate weight $(\mathrm{g})$ & $3417.01 \pm 507.03$ & $3518.75 \pm 445.54$ \\
\hline Neonate height $(\mathrm{cm})$ & $49.87 \pm 1.42$ & $50.28 \pm 0.66$ \\
\hline Apgar score at 1 minute & $9.89 \pm 0.94$ & $9.96 \pm 0.29$ \\
\hline Apgar score at 5 minutes & $9.91 \pm 0.92$ & 10 \\
\hline Premature delivery & $8(8.25 \%)$ & $2(5.71 \%)$ \\
\hline Fetal distress & $5(5.15 \%)$ & $1(2.86 \%)$ \\
\hline LBW & $5(5.15 \%)$ & $2(5.71 \%)$ \\
\hline Neonatal diseases & $5(5.15 \%)$ & $3(8.57 \%)$ \\
\hline Macrosomia & $15(15.46 \%)$ & $3(8.57 \%)$ \\
\hline
\end{tabular}

LBW: Low birth weight.

for the high prevalence of PPT in the TPOAb + TgAb + group [12]. However, the underlying mechanism remains unclear.

In the present study, 12-25\% women developed PPT as early as 6 weeks after delivery, while most of women in the four groups developed PPT at 3-6 months postpartum. In TPOAb-/TgAb-group, all PPT occurred within 6 months postpartum. Therefore, it is recommended that thyroid function test should be performed at 6 weeks postpartum and for women with antibody positive, it should be performed at 3 months at the latest in order to monitor early thyroid function changes. PPT can be biphasic (thyrotoxicosis followed by hypothyroidism) referred as classical form or monophasic (only thyrotoxicosis or only hypothyroidism). In this study, no significant difference was seen in the three forms of PPT between four groups up to 12 months postpartum. However, $80 \%$ PPT were presented as isolated hypothyroidism in TPOAb-/TgAb-group, while the number of classical forms, isolated thyrotoxicosis and isolated hypothyroidism women in other groups exhibited equally the same. Most of PPT in TPOAb + /TgAb-, TPOAb-/TgAb + and $\mathrm{TPOAb}+/ \mathrm{TgAb}+$ groups were followed up to 2 or further 3 years postpartum and presented as hypothyroidis, while in TPOAb-/ $\mathrm{TgAb}$ - group there presented more as euthyroidism. Compared to TPOAb-/TgAb- group, women in TPOAb + /TgAb + group were less likely to achieve euthyroidism 2 years postpartum. When it comes to 3 years postpartum, there existed fewer women as euthyroidism in TPOAb + /TgAb-, TPOAb-/TgAb + and TPOAb + /TgAb + group in comparison with TPOAb-/TgAb- group than 2 years postpartum. Higher levels of TPOAb and TgAb antibodies are associated with more postpartum thyroid injuries and more thyroid hormones releasing (behave as transient thyrotoxicosis), followed by hypothyroidism later over time. PPT can be regarded as a transient process of preexisting autoimmune thyroiditis which thyroid self-sensitization and subsequent thyroid cell destruction that may fluctuate over several years, gradually deteriorated toward hypothyroidism. Compared with the antibody positive groups, women who were all negative for TPOAb and TgAb had lower antibody levels and were likely to expose less damage to the thyroid, thus showing normal thyroid function postpartum. Despite our study did not see signif- icant difference between TPOAb positive and $\mathrm{TgAb}$ postive group, more efforts are needed to explore whether TgAb plays a similar role as TPOAb and how the two-antibody combined exert influence in the development of PPT. Given that PPT may be predictive of later failure in thyroid function, TPOAb or TgAb positive women in particular need close follow-up to improve postpartum life quality and prognosis.

Different studies have found the influence of TPOAb on maternal and neonatal adverse outcomes are controversial as mentioned before. Reports of associations between TgAb and adverse pregnancy outcomes are rare. A previous research found that there were no significant differences in maternal and neonatal outcomes except for PROM and low birth weight which were associated with TgAb and TPOAb, respectively [14]. Our study comprehensively analyzed the association between thyroid autoantibody status and pregnancy outcomes, and no association was found with antibody positivity and pregnancy outcomes including the mode of delivery, placenta previa, placental abruption, polyhydramnios, oligohydramnios, postpartum hemorrhage, preterm birth, fetal distress, low birth weight, neonatal disease, and macrosomia. Moreover, we found that premature rupture of membranes in TPOAb $+/ \mathrm{TgAb}-$ group was significantly higher than that in TPOAb-/TgAb- group, emphasizing the important role of TPOAb playing in pregnancy outcomes. Underlying mechanisms between TPOAb instead of $\mathrm{TgAb}$ and pregnancy outcomes especially PROM require to be further investigated.

In addition, thyroid function can be affected by stress, nutrition such as iodine, living style, etc. Despite the fact that overt and subclinical hypothyroidism can exert harmful effects on pregnancy, in our study, women diagnosed with overt and subclinical hypothyroidism were treated with levothyroxine as required to maintain normal thyroid function during pregnancy. In order to avoid the effect of iodine deficiency, we conducted this study in a population with adequate iodine. There were several limitations in our study. First, a larger number of subjects should be included. Second, the participants from multiple medical centers in different cities are needed. 
In conclusion, our study mainly analyzed the effects of TPOAb and $\mathrm{TgAb}$ on maternal and neonatal outcomes, indicating the high relevance between PROM and TPOAb. Also, the results showed that women who are TPOAb or TgAb positive were at a high risk for PPT, with the onset time at 6 weeks postpartum. Futhermore, we found that women with whatever TPOAb or TgAb positive were more likely to develop hypothyroidism at $2-3$ years postpartum. As a conquence, it is recommended that thyroid function reexamination should be regularly performed at 6 weeks postpartum in order to detect thyroid function changes as early as possible. More attention should be paid not only to TPOAb but also to TgAb in the underling mechanisms of thyroid function postpartum changes over time.

\section{Funding Information}

This research was supported by Maternal and Child Health Scientific Project of Jiangsu Province, China under Grant No. F201704; and Science and Education Revitalizing Health Program for Youth Science and Technology Project of Suzhou, China under Grant No. kjxw2018074.

\section{Conflict of Interest}

The authors declare that they have no conflict of interest.

\section{References}

[1] Yuan N, Sun J, Li Z et al. Relationship between anti-thyroid peroxidase antibody positivity and pregnancy-related and fetal outcomes in Euthyroid women: a single-center cohort study. BMC Pregnancy Childbirth 2020; 20: 491

[2] Yuan S, Yu N, Gao Y et al. Distribution of IgG subclasses of TgAb and TPOAb in sera from patients with Graves' disease, Graves' disease plus Hashimoto's thyroiditis and Hashimoto's thyrotoxicosis. Zhonghua Yi Xue Za Zhi 2014; 94: 110-114
[3] Hollowell JG, Staehling NW, Flanders WD et al. Serum TSH, T(4), and thyroid antibodies in the United States population (1988 to 1994): National Health and Nutrition Examination Survey (NHANES III). J Clin Endocrinol Metab 2002; 87: 489-499

[4] Negro R, Schwartz A, Gismondi R et al. Thyroid antibody positivity in the first trimester of pregnancy is associated with negative pregnancy outcomes. J Clin Endocrinol Metab 2011; 96: E920-E924

[5] Kuijpens JL, Vader HL, Drexhage HA et al. Thyroid peroxidase antibodies during gestation are a marker for subsequent depression postpartum. Eur J Endocrinol 2001; 145: 579-584

[6] Pop V], de Vries E, van Baar AL et al. Maternal thyroid peroxidase antibodies during pregnancy: a marker of impaired child development? J Clin Endocrinol Metab 1995; 80: 3561-3566

[7] Chen X, Jin B, Xia J et al. Effects of thyroid peroxidase antibody on maternal and neonatal outcomes in pregnant women in an iodinesufficient area in China. Int J Endocrinol 2016; 6461380

[8] Williams FL, Watson J, Ogston SA et al. Maternal and umbilical cord levels of T4, FT4, TSH, TPOAb, and TgAb in term infants and neurodevelopmental outcome at 5.5 years. J Clin Endocrinol Metab 2013; 98: 829-838

[9] Benvenga S. Targeted antenatal screening for predicting postpartum thyroiditis and its evolution into permanent hypothyroidism. Front Endocrinol (Lausanne) 2020; 11: 220

[10] Epp R, Malcolm J, Jolin-Dahel K et al. Postpartum thyroiditis. BM] 2021; 372: $\mathrm{n} 495$

[11] Bhattacharyya R, Mukherjee K, Das A et al. Anti-thyroid peroxidase antibody positivity during early pregnancy is associated with pregnancy complications and maternal morbidity in later life. J Nat Sci Biol Med 2015; 6: 402-405

[12] Li C, Zhou J, Huang $Z$ et al. The clinical value and variation of antithyroid antibodies during pregnancy. Dis Mrakers 2020; 8871951

[13] Ekinci El, Chiu WL, Lu ZX et al. A longitudinal study of thyroid autoantibodies in pregnancy: the importance of test timing. Clin Endocrinol (Oxf) 2015; 82: 604-610

[14] Chen LM, Zhang Q, Si GX et al. Associations between thyroid autoantibody status and abnormal pregnancy outcomes in euthyroid women. Endocrine 2015; 48: 924-928 